PROCEEDINGS OF THE

AMERICAN MATHEMATICAL SOCIETY

Volume 140, Number 7, July 2012, Pages 2333-2346

S 0002-9939(2011)11117-1

Article electronically published on November 17, 2011

\title{
ASYMPTOTIC DISTRIBUTIONS OF THE ZEROS OF A FAMILY OF HYPERGEOMETRIC POLYNOMIALS
}

\author{
JIAN-RONG ZHOU, H. M. SRIVASTAVA, AND ZHI-GANG WANG
}

(Communicated by Walter Van Assche)

\begin{abstract}
The main object of this paper is to consider the asymptotic distribution of the zeros of certain classes of the Gauss hypergeometric polynomials Some classical analytic methods and techniques are used here to analyze the behavior of the zeros of the Gauss hypergeometric polynomials,

$$
{ }_{2} F_{1}(-n, a ;-n+b ; z),
$$

where $n$ is a nonnegative integer. Owing to the connection between the classical Jacobi polynomials and the Gauss hypergeometric polynomials, we prove a special case of a conjecture made by Martínez-Finkelshtein, Martínez-González and Orive. Numerical evidence and graphical illustrations of the clustering of the zeros on certain curves are generated by Mathematica (Version 4.0).
\end{abstract}

\section{INTRODUCTION}

The celebrated Gauss hypergeometric function is defined by (see, for details, 1] and 22])

$$
{ }_{2} F_{1}\left[\begin{array}{rr}
\alpha, \beta ; & \\
\delta ; & z
\end{array}\right]=\sum_{k=0}^{\infty} \frac{(\alpha)_{k}(\beta)_{k}}{(\delta)_{k}} \frac{z^{k}}{k !} \quad(|z|<1),
$$

provided that no zeros appear in the denominator of (1.1). Here, as usual, $(\lambda)_{\nu}$ denotes the Pochhammer symbol or the shifted factorial, since

$$
(1)_{n}=n ! \quad(n \in \mathbb{N} ; \mathbb{N}:=\{1,2,3, \cdots\}),
$$

which is defined, in terms of the familiar Gamma function, by

$$
(\lambda)_{\nu}:=\frac{\Gamma(\lambda+\nu)}{\Gamma(\lambda)}= \begin{cases}1 & (\nu=0 ; \lambda \in \mathbb{C}), \\ \lambda(\lambda+1) \cdots(\lambda+n-1) & (\nu=n \in \mathbb{N} ; \lambda \in \mathbb{C}),\end{cases}
$$

with $\mathbb{C}$ being the set of complex numbers. It is understood (as usual) that $(0)_{0}:=1$.

If one or both of the numerator parameters $\alpha$ and $\beta$ is equal to a negative integer or zero, that is, if (for example)

$$
\alpha=-n \quad\left(n \in \mathbb{N}_{0}:=\{0,1,2, \cdots\}=\mathbb{N} \cup\{0\}\right),
$$

Received by the editors March 26, 2010 and, in revised form, August 10, 2010 and February 14, 2011

2010 Mathematics Subject Classification. Primary 33C05, 33C20; Secondary 30C15, 33C45.

Key words and phrases. Gauss hypergeometric function and polynomials, asymptotic distribution of zeros, zeros of ${ }_{2} F_{1}(-n, a ;-n+b ; z)$, Jacobi polynomials, hypergeometric reduction formulas, Euler-Mascheroni constant, Vitali's theorem, Hurwitz's theorem, Eneström-Kakeya theorem, hypergeometric identity, Mathematica (Version 4.0).

(C)2011 American Mathematical Society 
then the series in (1.1) terminates and reduces to a polynomial of degree $n$ in $z$. The natural question that arises in connection with any polynomials is the correlative properties of their zeros (see, for example, [5, 7, 8, 9, 10, 11, 21, 23, 24, 27]).

For the Gauss hypergeometric polynomials, the Euler integral representation, together with the saddle-point method, would generate the asymptotic zero distribution of some classes of ${ }_{2} F_{1}$ polynomials [2, 3, 13, 26]. On the basis of the connection between the Gauss ${ }_{2} F_{1}$ polynomial classes and the classical orthogonal Jacobi polynomials, a great deal of significant information about their zeros, including the location of zeros and the asymptotic zero distribution, has been obtained in [6]. Using different techniques which involve the direct investigation of the zero distribution of a ${ }_{3} F_{2}$ polynomial, the asymptotic behavior of the zeros of the polynomial ${ }_{2} F_{1}(-n, b ;-2 n ; z)$ for $b>0$ is obtained in 12 .

In the present paper, we propose to derive the asymptotic results for the zeros of the following hypergeometric ${ }_{2} F_{1}$ polynomials:

$$
\begin{gathered}
{ }_{2} F_{1}(-n, a ;-n+b ; z), \\
\left(a \in \mathbb{C} \backslash \mathbb{Z}_{0}^{-} ; b \in \mathbb{C} \backslash \mathbb{N}_{0}\right),
\end{gathered}
$$

where

$$
\mathbb{Z}_{0}^{-}=\{0,-1,-2, \cdots\}=\mathbb{Z}^{-} \cup\{0\} .
$$

By means of the hypergeometric identity (2.13) below, we also consider the asymptotic behavior of the zeros of the following Gauss hypergeometric polynomials:

$$
{ }_{2} F_{1}(-n, d ; f ; z) \text {. }
$$

In addition, the classical Jacobi polynomials $P_{n}^{(-n+l, s)}(z)$ and $P_{n}^{(s,-n+l)}(z)$, which are connected by the well-known relationship (3.1) with the Gauss hypergeometric polynomials, are also investigated. Numerical evidence and graphical illustrations of the clustering of the zeros on certain curves are generated by Mathematica (Version 4.0).

\section{A SET OF MAIN RESUlts}

For notational simplicity, we write

$$
{ }_{2} F_{1}\left[\begin{array}{c}
-n, a ; \\
-n+b ;
\end{array}\right]=: \sum_{k=0}^{n} a_{n, k} z^{k} \quad\left(a_{n, 0}:=1\right) .
$$

Lemma 1. Let

$$
a \in \mathbb{C} \backslash \mathbb{Z}_{0}^{-} ; b \in \mathbb{C} \backslash \mathbb{N}_{0}
$$

Then each of the following inequalities holds true:

$$
\left|a_{n, k}\right| \leqq \mathfrak{M}_{0} \cdot(k+1)^{|b|+|1-a|} \quad\left(0 \leqq k \leqq n ; n, k \in \mathbb{N}_{0}\right)
$$

where the constant $\mathfrak{M}_{0}>0$ depends on the parameters $a$ and $b$, but not on $n$ and $k$, and

$$
\left|\frac{a_{n, n-k}}{a_{n, n}}\right| \leqq \widetilde{\mathfrak{M}}_{0} \cdot(k+1)^{|b|+|1-a|} \quad\left(0 \leqq k \leqq n ; n, k \in \mathbb{N}_{0}\right),
$$

where the constant $\widetilde{\mathfrak{M}}_{0}>0$ depends on the parameters $a$ and $b$, but not on $n$ and $k$. 
Proof. We first consider the case when $0<k<n \quad(n, k \in \mathbb{N})$. Then, according to the following identity for the Pochhammer symbol $(\lambda)_{n}$ defined by (1.2):

$$
(-n)_{j}=: \begin{cases}\frac{(-1)^{j} n !}{(n-j) !} & \left(0 \leqq j \leqq n ; n, j \in \mathbb{N}_{0}\right), \\ 0 & \left(j \geqq n+1 ; n \in \mathbb{N}_{0} ; j \in \mathbb{N}\right),\end{cases}
$$

we have

$$
\frac{a_{n, k+1}}{a_{n, k}}=\frac{(n-k)(a+k)}{(n-b-k)(1+k)} .
$$

We also observe that

$$
\frac{n-k}{n-b-k}=1+\frac{b}{n-b-k}
$$

and

so that

$$
\frac{a+k}{1+k}=1+\frac{a-1}{1+k}
$$

and

$$
\ln \left(\frac{n-k}{|n-b-k|}\right) \leqq \frac{|b|}{|n-b-k|}
$$

Consequently, we have

$$
\ln \left(\frac{|a+k|}{1+k}\right) \leqq \frac{|a-1|}{1+k} .
$$

$$
\begin{aligned}
\ln \left|a_{n, k}\right| & =\ln \left(\prod_{l=0}^{k-1} \frac{\left|a_{n, l+1}\right|}{\left|a_{n, l}\right|}\right)=\sum_{l=0}^{k-1} \ln \left(\frac{\left|a_{n, l+1}\right|}{\left|a_{n, l}\right|}\right) \\
& \leqq \sum_{l=0}^{k-1}\left(\frac{|b|}{|n-b-l|}+\frac{|a-1|}{1+l}\right) \\
& \leqq \sum_{l=0}^{k-1}\left(\frac{|b|}{|n-b-k+1+l|}+\frac{|a-1|}{l+1}\right) \\
& \leqq \sum_{l=0}^{[|b|]} \frac{|b|}{|n-b-k+1+l|}+\sum_{l=0}^{k-1} \frac{|b|+|a-1|}{l+1} \\
& \leqq \mathfrak{M}+(|b|+|a-1|)[\ln (k+1)+\gamma],
\end{aligned}
$$

where $\mathfrak{M}>0$ is a constant and $\gamma$ is the Euler-Mascheroni constant given by

$\gamma:=\lim _{n \rightarrow \infty}\left(\sum_{k=1}^{n} \frac{1}{k}-\ln n\right) \cong 0.577215664901532860606512090082402431042 \cdots$.

It follows from (2.6) that

$$
\left|a_{n, k}\right| \leqq \mathfrak{M}_{0} \cdot(k+1)^{|b|+|a-1|} \quad(0<k<n ; n, k \in \mathbb{N}),
$$

where the constant $\mathfrak{M}_{0}>0$ depends on the parameters $a$ and $b$, but not on $n$ and $k$. The cases of the assertion (2.2) of Lemma 1 when $k=0$ and $k=n$ are trivial.

Next, by replacing $k$ by $n-k$ in (2.5), we have

$$
\frac{a_{n, n-k}}{a_{n, n-k+1}}=\frac{(k-b)(1+n-k)}{k(a+n-k)} .
$$


Since

it follows that

$$
\frac{k-b}{k}=1-\frac{b}{k} \quad \text { and } \quad \frac{1+n-k}{a+n-k}=1+\frac{1-a}{a+n-k},
$$

and

$$
\ln \left(\frac{|k-b|}{k}\right) \leqq \frac{|b|}{k}
$$

respectively. Therefore, we obtain

$$
\ln \left(\frac{1+n-k}{|a+n-k|}\right) \leqq \frac{|1-a|}{|a+n-k|},
$$

$$
\begin{aligned}
\ln \left(\frac{\left|a_{n, n-k}\right|}{\left|a_{n, n}\right|}\right) & =\ln \left(\prod_{l=1}^{k} \frac{\left|a_{n, n-l}\right|}{\left|a_{n, n-l+1}\right|}\right)=\sum_{l=1}^{k} \ln \left(\frac{\left|a_{n, n-l}\right|}{\left|a_{n, n-l+1}\right|}\right) \\
& \leqq \sum_{l=1}^{k}\left(\frac{|b|}{l}+\frac{|1-a|}{|a+n-l|}\right) \\
& \leqq \sum_{l=1}^{k}\left(\frac{|b|}{l}+\frac{|1-a|}{|a+n-k-1+l|}\right) \\
& \leqq \sum_{l=1}^{[|a|]+1} \frac{|1-a|}{|a+n-k-1+l|}+\sum_{l=1}^{k} \frac{|b|+|1-a|}{l} \\
& \leqq \mathfrak{N}+(|b|+|1-a|)[\ln (k+1)+\gamma],
\end{aligned}
$$

where $\mathfrak{N}>0$ is a constant and $\gamma$ is the Euler-Mascheroni constant given by (2.7). It follows from (2.8) that

$$
\frac{\left|a_{n, n-k}\right|}{\left|a_{n, n}\right|} \leqq \widetilde{\mathfrak{M}}_{0} \cdot(k+1)^{|b|+|1-a|},
$$

where the constant $\widetilde{\mathfrak{M}}_{0}>0$ depends on the parameters $a$ and $b$, but not on $n$ and $k$.

Finally, since the cases of assertion (2.3) of Lemma 1 when $k=0$ and $k=n$ are immediate, our proof of Lemma 1 is completed.

Theorem 1. For fixed parameters $a$ and $b$ constrained by

$$
a \in \mathbb{C} \backslash \mathbb{Z}_{0}^{-} \quad \text { and } \quad b \in \mathbb{C} \backslash \mathbb{N}_{0},
$$

the zeros of the Gauss hypergeometric polynomials,

$$
{ }_{2} F_{1}\left[\begin{array}{cc}
-n, a ; & z \\
-n+b ; &
\end{array}\right],
$$

approach the unit circle as $n \rightarrow \infty$.

Proof. From the assertion (2.2) of Lemma 1, it immediately follows that the sequence of polynomials

$$
\left\{\sum_{k=0}^{n} a_{n, k} z^{k}\right\}_{n \in \mathbb{N}_{0}}
$$

is uniformly bounded on the disk $\Omega$ given by

$$
\Omega=\{z: z \in \mathbb{C} \quad \text { and } \quad|z|<\rho \quad(0<\rho<1)\} .
$$


Furthermore, since (for fixed $k$ )

$$
a_{n, k}=\frac{(-n)_{k}(a)_{k}}{k !(-n+b)_{k}} \rightarrow \frac{(a)_{k}}{k !} \quad(n \rightarrow \infty)
$$

the sequence of functions

$$
\left\{\sum_{k=0}^{n} a_{n, k} z^{k}\right\}_{n \in \mathbb{N}_{0}}
$$

converges pointwise for $z(|z|<1)$ and, therefore, also uniformly, by Vitali's theorem [15, p. 252], to the sum

$$
\sum_{k=0}^{\infty} \frac{(a)_{k}}{k !} z^{k}={ }_{1} F_{0}(a ;-; z)=(1-z)^{-a} \quad(|z|<1 ; a \in \mathbb{C}) .
$$

Since the function $(1-z)^{-a}$ does not have any zeros inside the unit disk $|z|=1$, by Hurwitz's theorem [15, p. 205] there exists an index $N_{0}$ such that

$$
\sum_{k=0}^{n} a_{n, k} z^{k}
$$

does not have zeros on $\Omega$ for $n>N_{0}$. Hence there exist numbers $\rho_{n}$ constrained by

$$
0<\rho_{n}<1 \text { so that } \rho_{n} \rightarrow 1
$$

and we can ensure that

$$
\rho_{n} \geqq \rho \quad\left(n>N_{0} ; 0<\rho<1\right) .
$$

Next, from assertion (2.3) of Lemma 1, it also follows that the sequence of polynomials

$$
\frac{z^{n}}{a_{n, n}} \sum_{k=0}^{n} a_{n, k}\left(\frac{1}{z}\right)^{k}=\sum_{k=0}^{n} \frac{a_{n, n-k}}{a_{n, n}} z^{k}
$$

is uniformly bounded on $\Omega$. Moreover, since (for fixed $k$ )

$$
\frac{a_{n, n-k}}{a_{n, n}}=\frac{(-1)^{k} \Gamma(b) \Gamma(n+1) \Gamma(n+a-k)}{k ! \Gamma(b-k) \Gamma(n+a) \Gamma(n-k+1)} \rightarrow \frac{(1-b)_{k}}{k !} \quad(n \rightarrow \infty),
$$

the sequence of functions

$$
\left\{\sum_{k=0}^{n} \frac{a_{n, n-k}}{a_{n, n}} z^{k}\right\}_{n \in \mathbb{N}_{0}}
$$

converges pointwise for $z(|z|<1)$ and therefore also uniformly, by Vitali's theorem [15. p. 252], to the sum

$$
\sum_{k=0}^{\infty} \frac{(1-b)_{k}}{k !} z^{k}={ }_{1} F_{0}(1-b ;-; z)=(1-z)^{b-1} \quad(|z|<1 ; b \in \mathbb{C}) .
$$

Since the function $(1-z)^{b-1}$ does not have any zeros inside the unit disk $|z|=1$, by Hurwitz's theorem [15, p. 205] there exists an index $N_{0}$ such that

$$
\frac{z^{n}}{a_{n, n}} \sum_{k=0}^{n} a_{n, k}\left(\frac{1}{z}\right)^{k}
$$


does not have zeros on $\Omega$ for $n>N_{0}$; that is,

$$
\sum_{k=0}^{n} a_{n, k} z^{k}
$$

does not have zeros $|z|>\frac{1}{\rho}$. Hence there exist numbers $\gamma_{n}$ constrained by

$$
\gamma_{n}>1 \text { so that } \gamma_{n} \rightarrow 1
$$

and we can ensure that

$$
\gamma_{n} \leqq \frac{1}{\rho} \quad\left(n>N_{0} ; 0<\rho<1\right)
$$

Consequently, all zeros of the polynomial

$$
\sum_{k=0}^{n} a_{n, k} z^{k}
$$

lie in the annulus given by

$$
\left\{z: z \in \mathbb{C} \quad \text { and } \quad \rho_{n} \leqq|z| \leqq \gamma_{n}\right\}
$$

which completes the proof of Theorem 1.

Remark. In our proof of Theorem 1, we have used the fact that the sequences of functions

$$
\left\{\sum_{k=0}^{n} a_{n, k} z^{k}\right\}_{n \in \mathbb{N}_{0}} \quad \text { and } \quad\left\{\sum_{k=0}^{n} \frac{a_{n, n-k}}{a_{n, n}} z^{k}\right\}_{n \in \mathbb{N}_{0}}
$$

converge pointwise for $z(|z|<1)$ and therefore also uniformly, by Vitali's theorem [15, p. 252], to the sums

$$
\sum_{k=0}^{\infty} \frac{(a)_{k}}{k !} z^{k}={ }_{1} F_{0}(a ;-; z)=(1-z)^{-a} \quad(|z|<1 ; a \in \mathbb{C})
$$

and

$$
\sum_{k=0}^{\infty} \frac{(1-b)_{k}}{k !} z^{k}={ }_{1} F_{0}(1-b ;-; z)=(1-z)^{b-1} \quad(|z|<1 ; b \in \mathbb{C})
$$

respectively. Indeed, according to Lemma 1, we have

$$
\left|\sum_{k=0}^{n} a_{n, k} z^{k}\right| \leqq\left.\left.\left|\sum_{k=0}^{n}\right| a_{n, k}|\cdot| z\right|^{k}\left|\leqq \mathfrak{M}_{0} \cdot \sum_{k=0}^{n}(k+1)^{|b|+|1-a|}\right| z\right|^{k}
$$

Now, by applying d'Alembert's Ratio Test, we find that

$$
\lim _{k \rightarrow \infty}\left\{\frac{(k+2)^{|b|+|1-a|}|z|^{k+1}}{(k+1)^{|b|+|1-a|}|z|^{k}}\right\}=|z|,
$$

so that, clearly, the radius of convergence of the infinite series

$$
\sum_{k=0}^{\infty}(k+1)^{|b|+|1-a|}|z|^{k}
$$

is 1 and the sequence of polynomials

$$
\sum_{k=0}^{n}(k+1)^{|b|+|1-a|}|z|^{k}
$$


is uniformly bounded on the set $\Omega$ given by

$$
\Omega=\{z: z \in \mathbb{C} \text { and }|z|<\rho(0<\rho<1)\} .
$$

Consequently, the sequence of functions

$$
\left\{\sum_{k=0}^{n} a_{n, k} z^{k}\right\}_{n \in \mathbb{N}_{0}}
$$

converges pointwise for arbitrary $z_{0}\left(\left|z_{0}\right|<1\right)$. Similarly, the sequence of functions

$$
\left\{\sum_{k=0}^{n} \frac{a_{n, n-k}}{a_{n, n}} z^{k}\right\}_{n \in \mathbb{N}_{0}}
$$

can be seen to be convergent pointwise for arbitrary $z_{0} \quad\left(\left|z_{0}\right|<1\right)$. Thus, just as we claimed in our proof of Theorem 1, the sequences of functions

$$
\left\{\sum_{k=0}^{n} a_{n, k} z^{k}\right\}_{n \in \mathbb{N}_{0}} \quad \text { and } \quad\left\{\sum_{k=0}^{n} \frac{a_{n, n-k}}{a_{n, n}} z^{k}\right\}_{n \in \mathbb{N}_{0}}
$$

are pointwise convergent for all $z(|z|<1)$.

Theorem 2. If the fixed parameters $a$ and $b$ are constrained by

$$
0<a \leqq 1 \text { and } b<0,
$$

then the zeros of the Gauss hypergeometric polynomials

$$
{ }_{2} F_{1}\left[\begin{array}{cc}
-n, a ; & z \\
-n+b ; &
\end{array}\right]
$$

lie outside the unit disk $|z| \leqq 1$ and approach the unit circle $|z|=1$ as $n \rightarrow \infty$.

The proof of Theorem 2 is based upon the following classical result [18, p. 136].

Lemma 2 (Eneström-Kakeya Theorem [18, p. 136]). If

$$
0<a_{0}<a_{1}<\cdots<a_{n},
$$

then all zeros of the polynomial

$$
p(z)=a_{0}+a_{1} z+\cdots+a_{n} z^{n}
$$

lie in the unit disk $|z| \leqq 1$.

Proof of Theorem 2. According to Theorem 1, we only need to prove that the zeros of the Gauss hypergeometric polynomials

$$
{ }_{2} F_{1}(-n, a ;-n+b ; z)
$$

lie outside the unit circle $|z|=1$. We also find from (2.1) that

$$
z_{2}^{n} F_{1}\left[\begin{array}{cc}
-n, a ; & 1 \\
-n+b ; & z
\end{array}\right]=\sum_{k=0}^{n} a_{n, k} z^{n-k}=\sum_{k=0}^{n} a_{n, n-k} z^{k} .
$$

Thus, under the parametric constraints

$$
0<a \leqq 1 \text { and } b<0,
$$


which are already mentioned in the hypothesis of Theorem 2, we find from (2.5) that

$$
\frac{a_{n, n-(k+1)}}{a_{n, n-k}}=\frac{(k+1-b)(n-k)}{(k+1)(a+n-k-1)}>1 \quad(k=0,1, \cdots, n-1),
$$

which implies that the coefficients of the polynomial

$$
\widetilde{F}(z):=z_{2}^{n} F_{1}\left(-n, a ;-n+b ; z^{-1}\right)=\sum_{k=0}^{n} a_{n, n-k} z^{k}
$$

are positive and increasing, that is,

$$
0<a_{n, n}<a_{n, n-1}<\cdots<a_{n, 0} .
$$

It follows from Lemma 2 that the zeros of the polynomial $\widetilde{F}(z)$ defined by $(2.11)$ lie in unit disk $|z| \leqq 1$. Hence the zeros of

$$
{ }_{2} F_{1}(-n, a ;-n+b ; z)
$$

lie outside the unit disk $|z| \leqq 1$. This completes our proof of Theorem 2 .

Theorem 3. If the fixed parameters $a$ and $b$ are constrained by

$$
a \geqq 1 \text { and } 0<b<1 \text {, }
$$

then the zeros of the Gauss hypergeometric polynomials

$$
{ }_{2} F_{1}\left[\begin{array}{cc}
-n, a ; & z \\
-n+b ; &
\end{array}\right]
$$

lie inside the unit disk $|z| \leqq 1$ and approach the unit circle $|z|=1$ as $n \rightarrow \infty$.

Proof. By appealing appropriately to Theorem 1, we only need to prove that the zeros of

$$
{ }_{2} F_{1}(-n, a ;-n+b ; z)
$$

lie inside the unit disk $|z| \leqq 1$. Thus, under the parametric constraints

$$
a \geqq 1 \text { and } 0<b<1 \text {, }
$$

which are already mentioned in the hypothesis of Theorem 3, we find from (2.5) that

$$
\frac{a_{n, k+1}}{a_{n, k}}=\frac{(n-k)(a+k)}{(n-b-k)(1+k)}>1 \quad(k=0,1, \cdots, n-1),
$$

which implies that the coefficients of

$$
{ }_{2} F_{1}(-n, a ;-n+b ; z)
$$

are positive and increasing:

$$
0<a_{n, 0}<a_{n, 1}<\cdots<a_{n, n} .
$$

According to Lemma 2, we can see that the zeros of

$$
{ }_{2} F_{1}(-n, a ;-n+b ; z)
$$

lie inside the unit disk $|z| \leqq 1$. This completes the proof of Theorem 3 . 


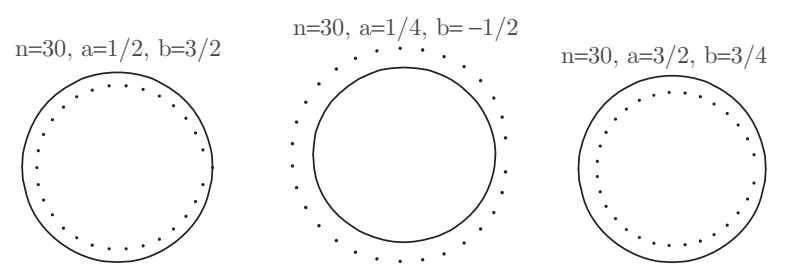

Figure 1. Zeros of ${ }_{2} F_{1}(-n, a ;-n+b ; z)$ and the unit circle $|z| \leqq 1$.

Figure 1 illustrates Theorems 1 to 3 with the help of the graphics in Mathematica (Version 4.0). It displays the zeros of the Gauss hypergeometric polynomials ${ }_{2} F_{1}(-n, a ;-n+b ; z)$ and their asymptotic curve $|z|=1$ for $n=30$ for various values of $a$ and $b$.

We shall make use of the following hypergeometric identity:

$$
{ }_{2} F_{1}\left[\begin{array}{rr}
-n, b ; & \\
c ; & z
\end{array}\right]=\frac{(c-b)_{n}}{(c)_{n}}{ }_{2} F_{1}\left[\begin{array}{rr}
-n, b ; & r \\
b-c-n+1 ; & 1-z
\end{array}\right]
$$

$\left(c \notin \mathbb{Z}_{0}^{-} ; c-b \notin \mathbb{Z}\right)$,

which is a rather immediate consequence of a familiar analytic continuation formula for the Gauss hypergeometric function in the form [14, p. 108, Equation 2.10(1)]

$$
{ }_{2} F_{1}(a, b ; c ; z)=\frac{\Gamma(c) \Gamma(c-a-b)}{\Gamma(c-a) \Gamma(c-b)}{ }_{2} F_{1}(a, b ; a+b-c+1 ; 1-z)
$$

$$
\begin{gathered}
+\frac{\Gamma(c) \Gamma(a+b-c)}{\Gamma(a) \Gamma(b)}(1-z)^{c-a-b}{ }_{2} F_{1}(c-a, c-b ; c-a-b+1 ; 1-z) \\
\left(|\arg (1-z)|<\pi ; c \notin \mathbb{Z}_{0}^{-} ; c-a-b \notin \mathbb{Z}\right)
\end{gathered}
$$

when we set $a=-n\left(n \in \mathbb{N}_{0}\right)$.

In view of (2.13) and Theorems 1 to 3, we have the following corollary.

Corollary 1. For fixed parameters $b$ and $c$ constrained by

$$
b \in \mathbb{C} \backslash \mathbb{Z}_{0}^{-}, \quad c \in \mathbb{C} \backslash \mathbb{Z}_{0}^{-} \quad \text { and } \quad c-b \in \mathbb{C} \backslash \mathbb{Z},
$$

all zeros of the hypergeometric polynomial ${ }_{2} F_{1}(-n, b ; c ; z)$ approach the circle $|z-1|=1$ as $n \rightarrow \infty$. Furthermore, if

$$
0<b \leqq 1 \text { and } 1+b<c
$$

then these zeros lie outside the disk $|z-1| \leqq 1$; and if

$$
b \geqq 1 \quad \text { and } \quad b<c<1+b,
$$

then these zeros lie inside the disk $|z-1| \leqq 1$.

Figure 2 illustrates Corollary 1 by showing the zeros of the hypergeometric polynomial ${ }_{2} F_{1}(-n, b ; c ; z)$ for $n=40$ and various values of $b$ and $c$ with their asymptotic curve $|z-1|=1$.

In the special case of Corollary 1 when $c=2 b$, Driver [6] obtained a more accurate result about the location of the zeros in Corollary 1 as follows. 

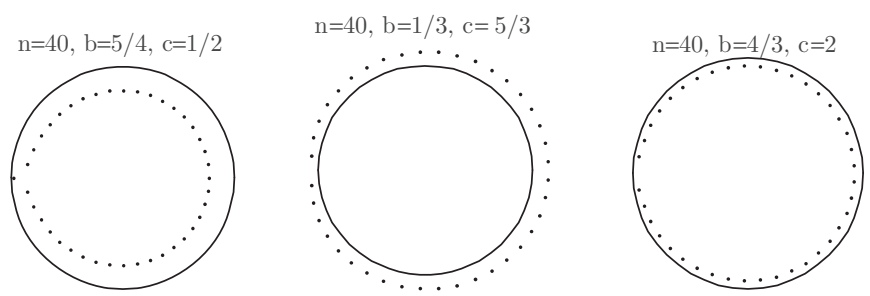

Figure 2. Zeros of ${ }_{2} F_{1}(-n, b ; c ; z)$ and the curve $|z-1|=1$.

Lemma 3 ([6, Theorem 1]). For each $b>-\frac{1}{2}$, all zeros of the hypergeometric polynomial $F(-n, b ; 2 b ; z)$ lie on the circle $|z-1|=1$.

From the identity (2.13) and Lemma 3, we have the following corollary.

Corollary 2. For fixed parameters a constrained by $a>-\frac{1}{2}$ and $a \neq 0$, the zeros of ${ }_{2} F_{1}(-n, a,-n+1-a ; z)$ lie on the unit circle $|z|=1$.

\section{RELATIONShiP With THE CLASSiCAL JaCOBI POLYNOMIALS}

The classical Jacobi polynomials $P_{n}^{(\alpha, \beta)}(z)$ have the following hypergeometric representation (see, for details, [25, Chapter 4]):

$$
(-1)^{n} P_{n}^{(\beta, \alpha)}(-z)=P_{n}^{(\alpha, \beta)}(z)=\frac{(1+\alpha)_{n}}{n !}{ }_{2} F_{1}\left[\begin{array}{rr}
-n, 1+\alpha+\beta+n ; & 1-z \\
1+\alpha ; &
\end{array}\right] .
$$

Our theorems can thus be applied to obtain asymptotic information about the zeros of the classical Jacobi polynomials (see also [4 for some recent results involving the Gauss hypergeometric polynomials and the classical Jacobi polynomials).

Theorem 4. (i) For fixed parameters $l$ and s constrained by

$$
l \in \mathbb{R} \backslash\left(\mathbb{N}_{0} \cup\{-1\}\right) \quad \text { and } \quad 1+l+s \in \mathbb{R} \backslash \mathbb{Z}_{0}^{-},
$$

all zeros of the Jacobi polynomials $P_{n}^{(-n+l, s)}(z)$ approach the circle $|z-1|=2$ as $n \rightarrow \infty$. Furthermore, if

$$
l<-1 \quad \text { and } \quad-1-l<s \leqq-l,
$$

then these zeros lie outside the disk $|z-1| \leqq 2$; and if

$$
-1<l<0 \quad \text { and } \quad s \geqq-l \text {, }
$$

then these zeros lie inside the disk $|z-1| \leqq 2$.

(ii) For fixed parameters $l$ and $s$ constrained by

$$
l \in \mathbb{R} \backslash\left(\mathbb{N}_{0} \cup\{-1\}\right) \quad \text { and } \quad 1+l+s \in \mathbb{R} \backslash \mathbb{Z}_{0}^{-},
$$

all zeros of the Jacobi polynomials $P_{n}^{(s,-n+l)}(z)$ approach the circle $|z+1|=2$ as $n \rightarrow \infty$. Furthermore, if

$$
l<-1 \quad \text { and } \quad-1-l<s \leqq-l,
$$

then these zeros lie outside the disk $|z+1| \leqq 2$; and if

$$
-1<l<0 \quad \text { and } \quad s \geq-l \text {, }
$$

then these zeros lie inside the disk $|z+1| \leqq 2$. 
Proof. (i) In view of the last member in (3.1), we can write (see also [25, p. 62])

$$
P_{n}^{(-n+l, s)}(z)=\frac{(1+l-n)_{n}}{n !}{ }_{2} F_{1}\left[\begin{array}{cc}
-n, 1+l+s ; & 1-z \\
-n+1+l ; &
\end{array}\right] .
$$

Also, upon setting

$$
a=1+l+s, \quad b=1+l \quad \text { and } \quad u=\frac{1-z}{2}
$$

in (3.2), we have

$$
P_{n}^{(-n+l, s)}(z)=\frac{(1+l-n)_{n}}{n !}{ }_{2} F_{1}\left[\begin{array}{rr}
-n, a ; & \\
-n+b ; & u
\end{array}\right] .
$$

By applying Theorem 1, we can readily deduce that all zeros of the Jacobi polynomials $P_{n}^{(-n+l, s)}(z)$ approach the circle

$$
\frac{|1-z|}{2}=|u|=1 \quad \text { as } \quad n \rightarrow \infty,
$$

provided that

$$
1+l+s=a \notin \mathbb{Z}_{0}^{-} \quad \text { and } \quad 1+l=b \notin \mathbb{N}_{0},
$$

that is, for fixed

$$
l \in \mathbb{R} \backslash\left(\mathbb{N}_{0} \cup\{-1\}\right) \text { and } 1+l+s \in \mathbb{R} \backslash \mathbb{Z}_{0}^{-} .
$$

Similarly, Theorems 2 and 3 can be applied to derive the remaining assertions of Theorem 4(i).

(ii) We now apply the symmetry relation in (3.1) in order to observe that

$$
P_{n}^{(s,-n+l)}(z)=(-1)^{n} P_{n}^{(-n+l, s)}(-z) .
$$

From (3.4) and the assertions of Theorem 4(i), we can establish the following fact. For fixed

$$
l \in \mathbb{R} \backslash\left(\mathbb{N}_{0} \cup\{-1\}\right) \quad \text { and } \quad 1+l+s \in \mathbb{R} \backslash \mathbb{Z}_{0}^{-},
$$

all zeros of the Jacobi polynomials $P_{n}^{(s,-n+l)}(z)$ approach the circle $|z+1|=2$ as $n \rightarrow \infty$. Furthermore, if

$$
l<-1 \quad \text { and } \quad-1-l<s \leqq-l,
$$

then these zeros lie outside the disk $|z+1| \leqq 2$; and if

$$
-1<l<0 \quad \text { and } \quad s \geqq-l,
$$

then these zeros lie inside the disk $|z+1| \leqq 2$. This proves the assertions of Theorem 4(ii), thereby completing the proof of Theorem 4.

Figure 3 and Figure 4 illustrate Theorem 4(i) and Theorem 4(ii), respectively. The zeros of the Jacobi polynomials $P_{n}^{(-n+l, s)}(z)$ and their asymptotic curve $|z-1|=2$ are shown in Figure 3. Figure 4 shows the zeros of the Jacobi polynomials $P_{n}^{(s,-n+l)}(z)$ for $n=50$ and various values of $s$ and $l$, together with their asymptotic curve $|z+1|=2$. 

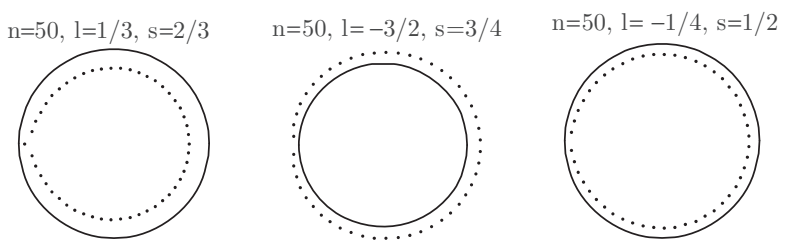

FigURE 3. Zeros of $P_{n}^{(-n+l, s)}(z)$ and the curve $|z-1|=2$.

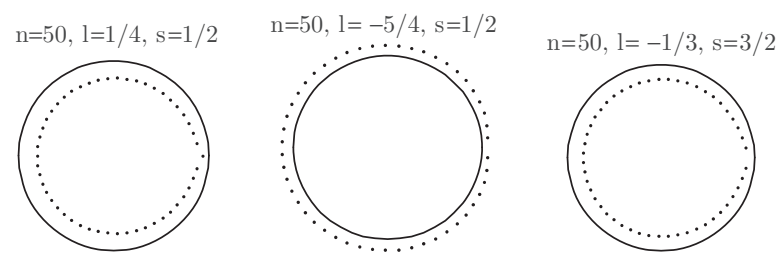

FiguRE 4. Zeros of $P_{n}^{(s,-n+l)}(z)$ and the curve $|z+1|=2$.

\section{Concluding Remarks AND OBSERvations}

It is interesting to note in conclusion that one of our results (Theorem 4 above) proves a special case of the conjecture made by Martínez-Finkelshtein et al. [19. Moreover, in [16, 17, 19, 20], the asymptotic distribution of the zeros of the Jacobi polynomials $P_{n}^{\left(\alpha_{n}, \beta_{n}\right)}(z)$ was investigated when

$$
\frac{\alpha_{n}}{n} \rightarrow A \quad \text { and } \quad \frac{\beta_{n}}{n} \rightarrow B \quad(n \rightarrow \infty),
$$

and the results presented here deal with the following two particular transitional cases:

$$
A+1=B=0 \quad \text { and } \quad A=B+1=0 .
$$

Finally, by applying (3.1) and Corollary 2, we can deduce Corollary 3.

Corollary 3. For fixed parameters $l$ constrained by $l<\frac{1}{2}(l \neq 0)$, all zeros of the Jacobi polynomials $P_{n}^{(-n+l,-1-2 l)}(z)$ lie on the circle $|z-1|=2$ and all zeros of the Jacobi polynomials $P_{n}^{(-1-2 l,-n+l)}(z)$ lie on the circle $|z+1|=2$.

\section{ACKNOWLEDGEMENTS}

The present investigation was supported by the National Natural Science Foundation of the People's Republic of China under Grant No. 11026205, the Provincial Natural Science Foundation of the People's Republic of China under Grant No. 10452800001004255, the Educational Commission of the Guangdong Province of the People's Republic of China under Grant No. LYM08101 and the Educational Commission of the Hunan Province of the People's Republic of China under Grant No. 08C118. 


\section{REFERENCES}

[1] W. N. Bailey, Generalized Hypergeometric Series, Cambridge Tracts in Mathematics and Mathematical Physics, No. 32, Cambridge University Press, Cambridge, London and New York, 1935. Reprinted by Stechert-Hafner Service Agency, New York and London, 1964. MR0185155 (32:2625)

[2] K. Boggs and P. Duren, Zeros of hypergeometric functions, Comput. Methods Funct. Theory 1 (2001), 275-287. MR.1931616 (2003k:33003)

[3] P. B. Borwein and W. Chen, Incomplete rational approximation in the complex plane, Constr. Approx. 11 (1995), 85-106. MR1323965 (95k:41024)

[4] K.-Y. Chen and H. M. Srivastava, A new result for hypergeometric polynomials, Proc. Amer. Math. Soc. 133 (2005), 3295-3302. MR2161152(2006d:33011)

[5] D. Dominici, K. Driver and K. Jordaan, Polynomial solutions of differential-difference equations, J. Approx. Theory 163 (2011), 41-48. MR2741218 (2011m:33019)

[6] K. Driver and P. Duren, Zeros of the hypergeometric polynomials $F(-n, b ; 2 b ; z)$, Indag. Math. (New Ser.) 11 (2000), 43-51. MR1809661 (2002d:33006)

[7] K. Driver and K. Jordaan, Zeros of ${ }_{3} F_{2}(-n, b, c ; d, e ; z)$ polynomials, Numer. Algorithms 30 (2002), 323-333. MR.1927508 (2003j:33018)

[8] K. Driver and K. Jordaan, Asymptotic zero distribution of ${ }_{3} F_{2}$ polynomials, Indag. Math. (New Ser.) 14 (2003), 319-327. MR2083078 (2005f:33004)

[9] K. Driver and K. Jordaan, Separation theorems for the zeros of certain hypergeometric polynomials, J. Comput. Appl. Math. 199 (2007), 48-55. MR.2267530 (2007m:33009)

[10] K. Driver and K. Jordaan, Pólya frequency sequences and real zeros of some ${ }_{3} F_{2}$ polynomials, J. Math. Anal. Appl. 332 (2007), 1045-1055. MR2324318 (2009m:33013)

[11] K. Driver, K. Jordaan and N. Mbuyi, Interlacing of zeros of linear combinations of classical orthogonal polynomials from different sequences, Appl. Numer. Math. 59 (2009), 2424-2429. MR2553144 (2011b:33017)

[12] K. Driver and M. Möller, Zeros of the hypergeometric polynomials $F(-n, b ;-2 n ; z)$, J. Approx. Theory 110 (2001), 74-87. MR1826086 (2002c:33001)

[13] P. Duren and B. J. Guillou, Asymptotic properties of zeros of hypergeometric polynomials, J. Approx. Theory 111 (2001), 329-343. MR.1849553 (2002f:33011)

[14] A. Erdélyi, W. Magnus, F. Oberhettinger and F. G. Tricomi, Higher Transcendental Functions, Vol. I, McGraw-Hill Book Company, New York, Toronto and London, 1953. MR0058756 (15:419i)

[15] E. Hille, Analytic Function Theory, Vol. II, Chelsea Publishing Company, Bronx, New York, 1973. MR0201608 (34:1490)

[16] A. B. J. Kuijlaars, A. Martínez-Finkelshtein, Strong asymptotics for Jacobi polynomials with varying nonstandard parameters, J. Analyse Math. 94 (2004), 195-234. MR 2124460 (2005k:33006)

[17] A. B. J. Kuijlaars, A. Martínez-Finkelshtein and R. Orive, Orthogonality of Jacobi polynomials with general parameters, Electron. Trans. Numer. Anal. 19 (2005), 1-17. MR2149265 (2006e:33010)

[18] M. Marden, Geometry of Polynomials, American Mathematical Society, Providence, Rhode Island, 1996. MR0225972 (37:1562)

[19] A. Martínez-Finkelshtein, P. Martínez-González and R. Orive, Zeros of Jacobi polynomials with varying nonclassical parameters, in: Special Functions (Hong Kong, 1999), World Scientific Publishing Company, River Edge, NJ (2000), 98-113. MR.1805976 (2002d:33017)

[20] A. Martínez-Finkelshtein and R. Orive, Riemann-Hilbert analysis for Jacobi polynomials orthogonal on a single contour, J. Approx. Theory 134 (2005), 137-170. MR2142296 (2006e:33013)

[21] P. Martínez-González and A. Zarzo, Higher order hypergeometric Lauricella function and zero asymptotics of orthogonal polynomials, J. Comput. Appl. Math. 233 (2010), 1577-1583. MR.2559348 (2011b:42092)

[22] H. M. Srivastava and P. W. Karlsson, Multiple Gaussian Hypergeometric Series, Halsted Press (Ellis Horwood Limited, Chichester), John Wiley and Sons, New York, Chichester, Brisbane and Toronto, 1985. MR834385 (87f:33015) 
[23] H. M. Srivastava and H. L. Manocha, A Treatise on Generating Functions, Halsted Press (Ellis Horwood Limited, Chichester), John Wiley and Sons, New York, Chichester, Brisbane and Toronto, 1984. MR750112 (85m:33016)

[24] H. M. Srivastava, J.-R. Zhou and Z.-G. Wang, Asymptotic distributions of the zeros of certain classes of hypergeometric functions and polynomials, Math. Comput. 80 (2011), 1769-1784. MR2785478

[25] G. Szegö, Orthogonal Polynomials, Fourth edition, American Mathematical Society Colloquium Publications, Vol. 23, American Mathematical Society, Providence, Rhode Island, 1975. MR0372517(51:8724)

[26] N. M. Temme, Large parameter cases of the Gauss hypergeometric function, J. Comput. Appl. Math. 153 (2003), 441-462. MR1985714 (2004f:33006)

[27] J.-R. Zhou and Y.-Q. Zhao, An infinite asymptotic expansion for the extreme zeros of the Pollaczek polynomials, Stud. Appl. Math. 118 (2007), 255-279. MR2305779 (2008e:33025)

Department of Mathematics, Foshan University, Foshan 528000, Guangdong Province, People's Republic of China

E-mail address: zhoujianrong2012@163.com

Department of Mathematics and Statistics, University of Victoria, Victoria, British Columbia V8W 3R4, Canada

E-mail address: harimsri@math.uvic.ca

School of Mathematics and Computing Science, Changsha University of Science and Technology (Yuntang Campus), Changsha 410114, Hunan Province, People's Republic OF CHINA

E-mail address: wangmath@163.com 